\title{
Imprints of coronal temperature disturbances on type III bursts
}

\author{
B. Li, I. H. Cairns, and P. A. Robinson
}

School of Physics, University of Sydney, New South Wales 2006, Australia

e-mail: boli@physics.usyd.edu.au

Received 17 December 2009 / Accepted 29 January 2010

\section{ABSTRACT}

\begin{abstract}
The electron temperature $T_{\mathrm{e}}$ and ion temperature $T_{\mathrm{i}}$ in the corona vary with time and location, due to transient and persistent activity on the Sun. The effects of spatially localized disturbances in $T_{\mathrm{e}}$ and $T_{\mathrm{i}}$ on coronal type III radio bursts are simulated. The disturbances are superimposed on monotonically varying temperature backgrounds and arise from spatially confined solar activity, Qualitatively and quantitatively different imprints are found on the curve of the maximum flux versus frequency of type III bursts, because of the disturbances in $T_{\mathrm{e}}$ and $T_{\mathrm{i}}$. The results indicate that nonthermal coronal type III bursts offer a new tool to probe and distinguish between spatially localized structures of $T_{\mathrm{e}}$ and $T_{\mathrm{i}}$ along the paths of type III beams. Furthermore, localized temperature disturbances may be responsible for some fine structures in type III bursts, e.g., striae in type IIIb bursts in the presence of multiple, localized temperature disturbances.
\end{abstract}

Key words. Sun: radio radiation - Sun: corona - methods: numerical

\section{Introduction}

The solar corona is highly dynamic and activity occurs both impulsively and continuously from small to large scales (e.g., microflares and flares). The activity results in energy injection and heat transfer, and so spatiotemporal variations in the temperature $T_{\mathrm{e}}$ and density $n_{\mathrm{e}}$ of electrons, and the ion temperature $T_{\mathrm{i}}$. For instance, spatially confined activity such as X-ray bright points may result in multiple, spatially localized disturbances in $T_{\mathrm{e}}$ and $T_{\mathrm{i}}$, as in Fig. 1. These may remain distinct from each other because tangential discontinuities can separate different thermal plasmas for long periods. In addition, $T_{\mathrm{i}}$ disturbances may be more localized and last much longer than $T_{\mathrm{e}}$ disturbances, because ions have much lower thermal speeds than electrons. Knowledge of the spatial profiles of coronal temperatures is usually obtained by using visible, UV, X-ray, and thermal radio emission. This knowledge, currently inadequate, is vital to understanding coronal heating and solar wind acceleration.

Type III solar radio bursts are produced when energetic electrons accelerated in flares propagate as beams along open magnetic field lines into the interplanetary (IP) medium, and drive Langmuir $(L)$ waves near the electron plasma frequency $f_{\mathrm{p}}$ and radiation near $f_{\mathrm{p}}$ and/or $2 f_{\mathrm{p}}$. Coronal type IIIs are one of the most important diagnostics of electron acceleration in flares and coronal conditions (Aschwanden 2002, 2006). For example, coronal type IIIs have been used to extract the Sun's density profile, and solar-wind-like regions with $n_{\mathrm{e}} \propto\left(r-R_{\odot}\right)^{-2}$ are found to be common below $2 R_{\odot}$, where $R_{\odot}$ is the solar radius (Cairns et al. 2009).

Sometimes, fine and/or sudden intensity changes in coronal type IIIs are observed (Fomichev \& Chertok 1977; Suzuki $\&$ Dulk 1985). For example, type IIIb (stria) bursts show non-smooth variations in flux versus frequency, and chains of narrow-band structures are caused by successive modulations in flux (de La Noe \& Boischot 1972). It has been variously conjectured that the flux modulations in type IIIb bursts occur because of modulational instability related to the dynamics of

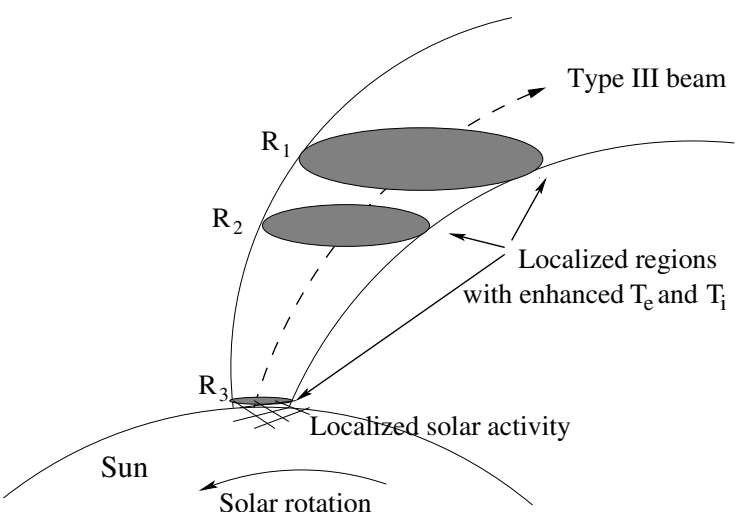

Fig. 1. Illustration of the production of localized regions with enhanced coronal temperatures in an open magnetic flux tube, through which a type III beam propagates (viewed from above the north pole). Localized regions $R_{1}-R_{3}$ are caused by three separate episodes of activity at localized solar source, with the latest activity producing $R_{3}$. For clarity, the flux tube is assumed to have a regular spiral shape.

beam and $L$ waves (Smith \& de La Noe 1976), or filamentary coronal density structures (Takakura \& Yousef 1975).

The primary aim of this letter is to demonstrate numerically the effects on coronal type IIIs of spatially localized disturbances of $T_{\mathrm{e}}$ and $T_{\mathrm{i}}$. Our simulations show that the dynamic spectrum of $2 f_{\mathrm{p}}$ emission is modulated at frequencies corresponding to the disturbances; so is the $f_{\mathrm{p}}$ emission, although the predictions are generally below observable levels. Importantly, distinct imprints of the $T_{\mathrm{e}}$ and $T_{\mathrm{i}}$ disturbances exist on the curve of the maximum flux versus frequency for the $2 f_{\mathrm{p}}$ emission. The second aim is to show that detailed structures in coronal type IIIs can be used to probe and ideally differentiate between localized variations of $T_{\mathrm{e}}$ and $T_{\mathrm{i}}$ along the beam path. Fine structures resembling those in type IIIb bursts are produced by multiple temperature disturbances, which suggests that localized temperature disturbances may lead to type IIIb bursts. 


\section{Numerical model}

The spatial variation in the ion temperature is defined by $T_{\mathrm{i}}=$ $T_{\mathrm{i}}(x)$ at time $t=0$, where $x$ is along the radial direction and represents the distance above the photosphere. Since we assume that ions remain Maxwellian and unchanged during the simulations, as in our earlier work (Li et al. 2008a, 2008b), $T_{\mathrm{i}}$ retains its original profile at all times. However, for an inhomogeneous plasma with position-dependent electron temperature $T_{\mathrm{e}}(x)$ and/or density $n_{\mathrm{e}}(x)$, a source term needs to be added to the kinetic equation for electrons to preserve the given $T_{\mathrm{e}}(x)$ and $n_{\mathrm{e}}(x)$ profiles for simulation periods longer than the propagation time of a beam traversing the simulation domain. The requirement to have a source term arises because we allow electrons to evolve dynamically. Specifically, the electron distribution function $f_{\mathrm{e}}(t, x, v)$ evolves following (Li et al. 2002):

$\frac{\partial f_{\mathrm{e}}}{\partial t}+v \frac{\partial f_{\mathrm{e}}}{\partial x}=\frac{\partial}{\partial v}\left(A f_{\mathrm{e}}\right)+\frac{\partial}{\partial v}\left(D \frac{\partial f_{\mathrm{e}}}{\partial v}\right)$

where $v$ denotes electron speed. The first and second terms on the right-hand side (r.h.s.) of Eq. (1) represent spontaneous and induced emission, respectively, and the coefficient $D$ describes the coupling between the beam and $L$ waves. The density is given by $n_{\mathrm{e}}=\int f_{\mathrm{e}}(v) \mathrm{d} v$.

Under thermal and steady state conditions, $f_{\mathrm{e}}$ is Maxwellian and given by $f_{\theta}=n_{\mathrm{e}}\left(m_{\mathrm{e}} / 2 \pi k_{\mathrm{B}} T_{\mathrm{e}}\right)^{1 / 2} \exp \left(-m_{\mathrm{e}} v^{2} / 2 k_{\mathrm{B}} T_{\mathrm{e}}\right)$, where $m_{\mathrm{e}}$ is the electron mass. The r.h.s. of Eq. (1) then vanishes because of the self-consistent definitions of $A$ and $D$ (Li et al. 2002). However, the left-hand side (1.h.s.) of Eq. (1) reduces to the advection term $v \partial f_{\theta} / \partial x$, which is non-vanishing for plasmas with $x$-dependent $T_{\mathrm{e}}$ and/or $n_{\mathrm{e}}$. So a source term is required for Eq. (1) to remain self-consistent. We thus add a source term $S(x, v)$ to the r.h.s. of Eq. (1), and set

$$
\begin{aligned}
S(x, v) & =v \frac{\partial}{\partial x} f_{\theta}\left(n_{\mathrm{e}}, T_{\mathrm{e}}, v\right), \\
& =v f_{\theta}\left(n_{\mathrm{e}}, T_{\mathrm{e}}, v\right)\left[\frac{\partial}{\partial x} \ln n_{\mathrm{e}}(x)+\frac{1}{2}\left(\frac{v^{2}}{v_{\mathrm{e}}^{2}}-1\right) \frac{\partial}{\partial x} \ln T_{\mathrm{e}}(x)\right],
\end{aligned}
$$

where $v_{\mathrm{e}}=\left(k_{\mathrm{B}} T_{\mathrm{e}} / m_{\mathrm{e}}\right)^{1 / 2}$ is the electron thermal speed. Equation (3) consists of two terms, which correspond to inhomogeneities in $n_{\mathrm{e}}$ and $T_{\mathrm{e}}$, respectively, as discussed below.

We consider first the case in which the plasma has varying $n_{\mathrm{e}}$ but uniform $T_{\mathrm{e}}$, for which only the first term of Eq. (3) remains. For $n_{\mathrm{e}}(x)$ monotonically increasing (decreasing) with increasing $x, S>0(S<0)$ for electrons with $v>0$, and so acts as a source (sink). In contrast, $S$ is a sink (source) for electrons with $v<0$. The physics of the case when $n_{\mathrm{e}}(x)$ decreases monotonically is as follows. On the one hand, for $v>0$ more electrons move into rather than out of a given coronal layer during a finite time interval. Thus, to maintain the given $n_{\mathrm{e}}$ and $T_{\mathrm{e}}$ profiles a sink is needed to remove the excess electrons. On the other hand, for $v<0$ more electrons move out of than into the given layer, so a source is required to supply the electrons lost because of the imbalance.

When $T_{\mathrm{e}}$ is nonuniform but $n_{\mathrm{e}}$ is homogeneous, only the second term of Eq. (3) remains. The nature (source or sink) of the $S$ term now depends on both the trend of the $T_{\mathrm{e}}$ profile and the electron speed, via the factors $\partial\left(\ln T_{\mathrm{e}}\right) / \partial x$ and $v\left[\left(v / v_{\mathrm{e}}\right)^{2}-1\right]$, respectively. If we assume that $T_{\mathrm{e}}$ monotonically increases with $x$, then at a given coronal layer statistically more electrons with $v>v_{\mathrm{e}}$ move outward rather than inward. To maintain the given $T_{\mathrm{e}}$ and $n_{\mathrm{e}}$ profiles, a source is therefore needed to supply the loss of electrons caused by the imbalance. In contrast, at the

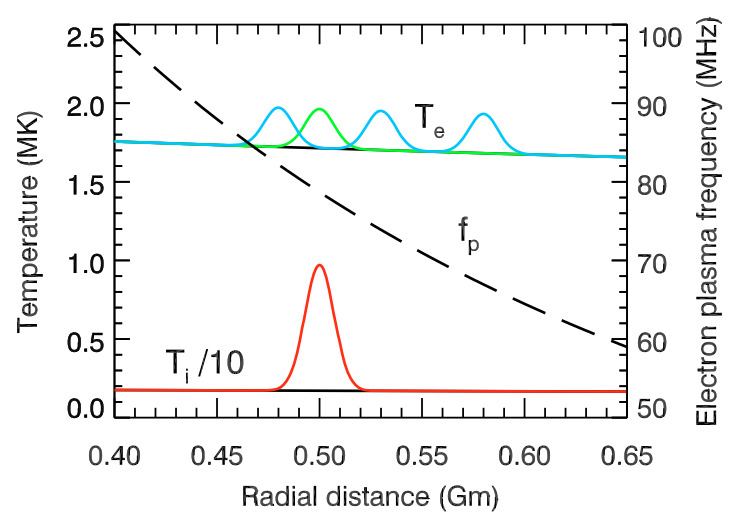

Fig. 2. Spatial profiles of $T_{\mathrm{e}}, T_{\mathrm{i}}$, and $f_{\mathrm{p}}$ in the simulations, with $T_{\mathrm{e}}$ and $T_{\mathrm{i}}$ given by Eq. (4). ( $\left.\mathcal{A}\right)$ Solid black: monotonic variations of $T_{\mathrm{e}}$ and $T_{\mathrm{i}} / 10$ with $T_{\mathrm{m}}^{\mathrm{e}}=T_{\mathrm{m}}^{\mathrm{i}}=2 \mathrm{MK}$. $(\mathcal{B})$ Green: background $T_{\mathrm{e}}$ in $\mathcal{A}$ with a superposed $T_{\mathrm{e}}$ disturbance with $T_{1}^{\mathrm{e}}=0.25 \mathrm{MK}, x_{1}^{\mathrm{e}}=0.5 \mathrm{Gm}$, and $\Delta x_{1}^{\mathrm{e}}=$ $0.01 \mathrm{Gm} .(C)$ Red: $T_{\mathrm{i}} / 10$ for a localized $T_{\mathrm{i}}$ disturbance with $T_{1}^{\mathrm{i}}=8 \mathrm{MK}$, $x_{1}^{\mathrm{i}}=0.5 \mathrm{Gm}$, and $\Delta x_{1}^{\mathrm{i}}=0.01 \mathrm{Gm}$ superposed on $\mathcal{A}$ 's background $T_{\mathrm{i}}$. $(\mathcal{D})$ Blue: $T_{\mathrm{e}}$ for three localized $T_{\mathrm{e}}$ disturbances with $x_{1}^{\mathrm{e}}=0.48 \mathrm{Gm}$, $x_{2}^{\mathrm{e}}=0.53 \mathrm{Gm}, x_{3}^{\mathrm{e}}=0.58 \mathrm{Gm}, \Delta x_{l}^{\mathrm{e}}=0.01 \mathrm{Gm}$, and $T_{l}^{\mathrm{e}}=0.25 \mathrm{MK}$ $(l=1-3)$ added to the background $T_{\mathrm{e}}$ in $\mathcal{A}$. The $f_{\mathrm{p}}$ curve (dashed black) is for the 10-fold Baumbach-Allen model.

same layer more slow electrons with $0<v<v_{\mathrm{e}}$ move in rather than out, thus the excess electrons need to be drained away to retain the balance.

In general, both terms in Eq. (3) remain for arbitrary, inhomogeneous profiles of $n_{\mathrm{e}}$ and $T_{\mathrm{e}}$. In the corona various effects may contribute to the source $S$. For instance, small-scale activity, e.g., jets, can heat and transport electrons between different locations, causing either gain or loss of electrons at these locations.

The simulations of coronal type IIIs use here our quasilinearbased model for plasma emission (Li et al. 2008a, 2008b). The model includes a 3D source region with stratified 2D layers that vary with $x$, the dynamics of beam, $L$, ion-sound $(S)$, and transverse waves coupled via quasilinear and nonlinear processes, and radiation propagation from the source to a remote observer. The model is generalized by including the term $S$ in Eq. (1) which allows us to study the effects of localized temperature variations.

\section{Simulation results}

We assume that the coronal temperatures follow

$T_{s}(x)=T_{\mathrm{m}}^{s}\left(1+\frac{x}{R_{\odot}}\right)^{-2 / 7}+\sum_{l} T_{l}^{s} \exp \left[-\left(\frac{x-x_{l}^{s}}{\Delta x_{l}^{s}}\right)^{2}\right]$,

where $s=\mathrm{e}$ and $\mathrm{i}$ are for electrons and ions, respectively. The first and second terms represent monotonic variations and localized disturbances $(l \geq 1)$, respectively. For $T_{\mathrm{e}}$, the monotonic term is for quiet coronal conditions (Parker 2007). Each localized component is Gaussian and centered on $x_{l}^{s}$ with a characteristic width $\Delta x_{l}^{s}$ and a peak temperature $T_{l}^{s}$.

Figure 2 shows the spatial profiles of $T_{\mathrm{e}}$ and $T_{\mathrm{i}}$ in the simulations. Four different temperature conditions are considered: $(\mathcal{A})$ both $T_{\mathrm{e}}$ and $T_{\mathrm{i}}$ decrease monotonically; $(\mathcal{B})$ a localized $T_{\mathrm{e}}$ disturbance is imposed on the background $T_{\mathrm{e}}$ in $\mathcal{A}$, and $T_{\mathrm{i}}$ is as in $\mathcal{A}$; (C) a localized $T_{\mathrm{i}}$ disturbance is imposed on the background $T_{\mathrm{i}}$ in $\mathcal{A}$, and $T_{\mathrm{e}}$ is as in $\mathcal{A} ;(\mathcal{D})$ three localized $T_{\mathrm{e}}$ disturbances are added to the background $T_{\mathrm{e}}$ in $\mathcal{A}$, and $T_{\mathrm{i}}$ is as in $\mathcal{A}$. We assume that $T_{1}^{\mathrm{i}}=8 \mathrm{MK}$ for case $C$, which is much higher than the 
B. Li et al.: Imprints of coronal temperature disturbances on type III bursts

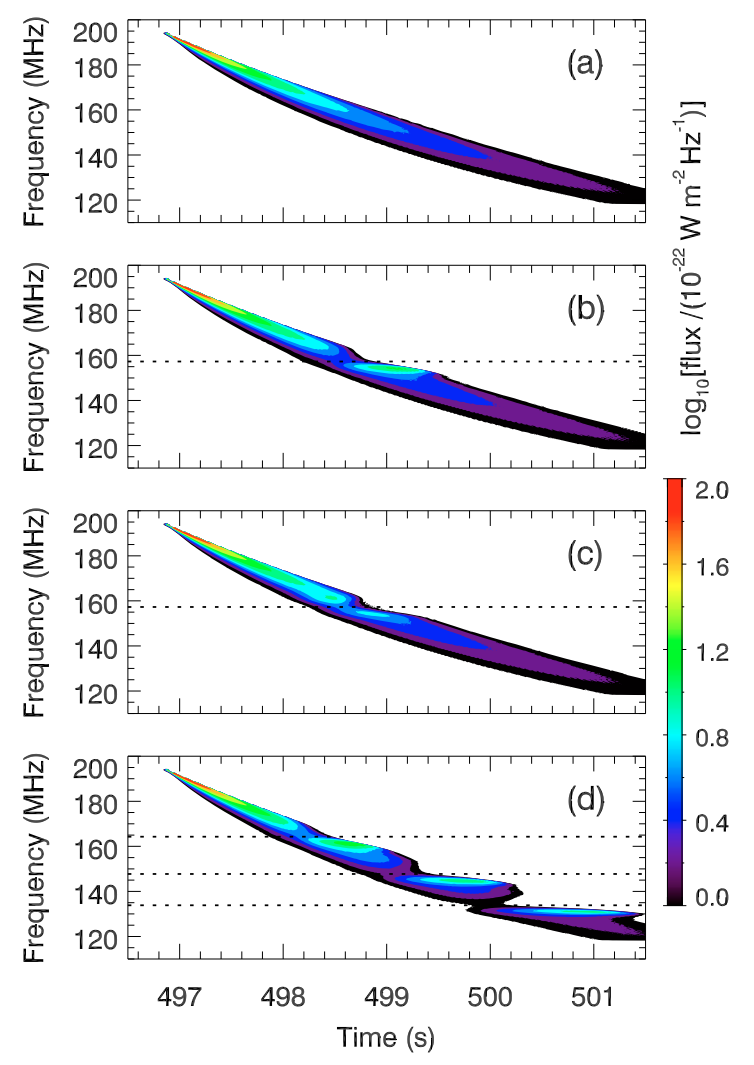

Fig. 3. Simulated $2 f_{\mathrm{p}}$ emission spectrum observed at Earth under the four temperature profiles in Fig. 2: a) case $\mathcal{A}, \mathbf{b})$ case $\mathcal{B}$, c) case $C$, and d) case $\mathcal{D}$. Dotted lines in b)-d) indicate $f=2 f_{\mathrm{p}}\left(x_{l}^{s}\right)$ at the central location $x_{l}^{s}$ of the temperature disturbances.

value of $T_{l}^{\mathrm{e}}=0.25 \mathrm{MK}$ for cases $\mathcal{B}$ and $\mathcal{D}$. The $T_{l}^{s}$ parametrization is based upon observations (e.g., Kohl et al. 1996), which show much higher coronal proton temperatures than $T_{\mathrm{e}}$, reaching 4-6 MK or higher at height $x \approx 1 R_{\odot}$. Here we choose a higher $T_{\mathrm{i}}$ than observed so far to demonstrate clearly the effects of $T_{\mathrm{i}}$ localization.

We assume the 10-fold Baumbach-Allen model (Baumbach 1937; Allen 1947) for $n_{\mathrm{e}}$, and Fig. 2 also shows the resulting $f_{\mathrm{p}}$ profile. The acceleration of electrons during flares is represented by adding a heating term $H$ (Robinson \& Benz 2000; $\mathrm{Li}$ et al. 2002) to the r.h.s. of Eq. (1): $H=F_{\mathrm{h}}\left[f_{\theta}\left(T_{\mathrm{h}}\right)-\right.$ $\left.f_{\theta}\left(T_{\mathrm{e}}\right)\right] \exp \left[-\left(t-t_{\mathrm{h}}\right)^{2} /(\delta t)^{2}-\left(x-x_{\mathrm{h}}\right)^{2} /(\delta x)^{2}\right] /(\sqrt{\pi} \delta t)$. Here a fraction $F_{\mathrm{h}}$ of electrons are heated from $T_{\mathrm{e}}$ to $T_{\mathrm{h}}\left(>T_{\mathrm{e}}\right)$ over a typical region $(\delta t, \delta x)$ centered at $\left(t_{\mathrm{h}}, x_{\mathrm{h}}\right)$. Based on observations (Aschwanden 2002; Klein et al. 2005), we choose $F_{\mathrm{h}}=10^{-5}$, $T_{\mathrm{h}}=25 \mathrm{MK}, t_{\mathrm{h}}=50 \mathrm{~ms}, \delta t=2 \mathrm{~ms}, x_{\mathrm{h}}=0.405 \mathrm{Gm}$, and $\delta x=1 \mathrm{Mm}$. Other simulation parameters are as in $\mathrm{Li}$ et al. (2008a).

Figures 3 and 4 show the dynamic spectrum of $2 f_{\mathrm{p}}$ emission at Earth and the corresponding maximum flux $\mathcal{F}(f)$ versus frequency $f$, respectively, under the four different temperature conditions in Fig. 2. The $f_{\mathrm{p}}$ emission (not shown) is too weak, with flux $\ll 1$ solar flux unit $(\mathrm{sfu})=10^{-22} \mathrm{~W} \mathrm{~m}^{-2} \mathrm{~Hz}^{-1}$, to be observable except for cases $C$ and $\mathcal{D}$. This is due primarily to strong free-free absorption, strong radiation loss by scattering, and weak source emission (Robinson \& Benz 2000; Li et al. 2008a). Below we discuss mainly the $2 f_{\mathrm{p}}$ results.

First, Figs. 3a and 4 (black curve) show that for case $\mathcal{A}$ the spectrum varies smoothly, and $\mathcal{F}$ decreases with $f$ after radiation onset, respectively. These properties and other spectral

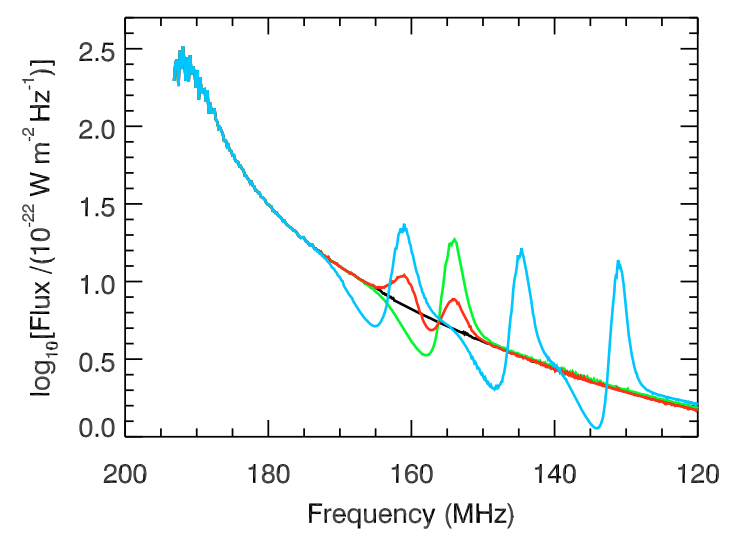

Fig. 4. Maximum $2 f_{\mathrm{p}}$ flux versus frequency for the four temperature profiles in Fig. 2: cases $\mathcal{A}$ (black), $\mathcal{B}$ (green), $C$ (red), and $\mathcal{D}$ (blue).

characteristics, e.g., drift rate (not shown), agree quantitatively with typical observations, as in our previous work ( $\mathrm{Li}$ et al. 2008a).

Figure $3 \mathrm{~b}$ shows the $2 f_{\mathrm{p}}$ spectrum for case $\mathcal{B}$. The spectrum is modulated at $f$ corresponding to the $T_{\mathrm{e}}$ disturbance, and the flux is suppressed close to the center of the disturbance. Figure 4 (green curve) illustrates fine structures in $\mathcal{F}$ caused by the disturbance. Specifically, as $f$ varies from high to low frequencies $\mathcal{F}$ varies according to the sequence "same $\rightarrow$ decrease $\rightarrow$ increase $\rightarrow$ same", relative to case $\mathcal{A}$. In addition, $\mathcal{F}$ is modulated within the range $\approx 150-165 \mathrm{MHz}$, corresponding to the disturbed region between $x_{1}^{\mathrm{e}}+2 \Delta x_{1}^{\mathrm{e}}$ and $x_{1}^{\mathrm{e}}-2 \Delta x_{1}^{\mathrm{e}}$. Furthermore, $\mathcal{F}$ reaches a local minimum and maximum at $f \approx 158 \mathrm{MHz} \approx$ $2 f_{\mathrm{p}}\left(x_{1}^{\mathrm{e}}\right)=157 \mathrm{MHz}$ and $\approx 154 \mathrm{MHz}$, decreasing and increasing by factors of $\approx 1.8$ and $\approx 3.8$ relative to case $\mathcal{A}$, respectively.

Physically, on entering the region with increasing $T_{\mathrm{e}}$, the beam becomes relatively narrower and slightly faster because $f_{\theta}$ extends to higher $v$. This causes the weaker growth of $L$ waves, mainly because less free energy is available, although the collisional damping rate $\left(\propto T_{\mathrm{e}}^{-3 / 2}\right)$ of $L$ waves decreases slightly (Li et al. 2008b). The source $2 f_{\mathrm{p}}$ emission is thus weaker, so is the remote radiation, although loss by free-free absorption is reduced locally because $T_{\mathrm{e}}$ increases. On passing the central region of the disturbance, the beam becomes relatively wider and slower because $T_{\mathrm{e}}$ decreases. The combined effects of more free energy now being available and the lower collisional damping rate result in stronger $L$ waves, and so stronger source $2 f_{\mathrm{p}}$ emission. Remote radiation is further strongly enhanced because of the locally weaker free-free absorption. On exiting the disturbed region, the beam, source waves, and radiation recover their properties for case $\mathcal{A}$.

Figure $3 \mathrm{c}$ shows the $2 f_{\mathrm{p}}$ spectrum for case $C$ with a single localized $T_{\mathrm{i}}$ disturbance. The spectrum appears qualitatively similar to that in Fig. 3b in terms of spectral modulation. However, detailed examination of the variation in $\mathcal{F}$ versus $f$ in Fig. 4 (red curve) shows both qualitative and quantitative differences and similarities exist relative to case $\mathcal{B}$, as follows:

1. The sequence of $\mathcal{F}$ versus $f$ from high to low frequencies relative to case $\mathcal{A}$ is "same $\rightarrow$ increase $\rightarrow$ decrease $\rightarrow$ increase $\rightarrow$ same", which is distinct from that for case $\mathcal{B}$.

2. The frequencies $\approx 161 \mathrm{MHz}$ and $154 \mathrm{MHz}$ of the two local peaks, and $\approx 157 \mathrm{MHz}$ at the center of the local dip differ evidently from, and is quantitatively similar to, those of the single peak and trough for case $\mathcal{B}$, respectively.

3. The quantitative modulation effects on $\mathcal{F}$ caused by the $T_{\mathrm{i}}$ disturbance are weaker than those caused by the $T_{\mathrm{e}}$ 
disturbance. For instance, $\mathcal{F}$ increases by a factor $\approx 1.5$ at the two local peaks relative to case $\mathcal{A}$. This factor is smaller than the factor $\approx 3.8$ for case $\mathcal{B}$, where $T_{\mathrm{e}}$ increases locally by $\lesssim 15 \%$, while $T_{\mathrm{i}}$ here increases locally by a factor $\lesssim 2.6$.

4. The frequency region of the $\mathcal{F}$ modulation is basically the same as for case $\mathcal{B}$.

The $2 f_{\mathrm{p}}$ results for cases $\mathcal{B}$ and $C$ differ because varying $T_{\mathrm{i}}$ has different effects from varying $T_{\mathrm{e}}$. Specifically, varying $T_{\mathrm{i}}$ does not affect radiation propagation but affects $L$ waves by changing the rate of the nonlinear Langmuir electrostatic (ES) decay, which generates product $L$ and $S$ waves, thereby changing the rate of $2 f_{\mathrm{p}}$ emission ( $\mathrm{Li}$ et al. 2005, 2008a).

The trend of $\mathcal{F}$ versus $f$ for case $C$ in Fig. 4 is caused by the following. As the beam enters the region where $T_{\mathrm{i}}$ increases, the product $L$ waves become stronger because of the stronger ES decay, leading to stronger $2 f_{\mathrm{p}}$ source emission (produced by coupling between primary and product $L$ waves), hence stronger remote emission ( $\mathrm{Li}$ et al. 2005). However, as $T_{\mathrm{i}}$ increases further, the level of primary $L$ waves decreases because the even stronger decay process transfer the primary $L$ wave energy into the product $L$ waves (Li et al. 2003). The forward-directed (antiSunward) emission is thus weaker. Consequently, a local dip in $\mathcal{F}$ appears at frequencies corresponding to where $T_{\mathrm{i}}$ maximizes. In the simulations, we neglected the backward-directed $2 f_{\mathrm{p}}$ emission, which is generated by coupling between product $L$ waves (Li et al. 2008a). In general, the backward emission contributes negligibly to $\mathcal{F}$ because the radiation is partly lost and time-delayed by strong scattering and reflection closer to the Sun and undergoes additional free-free absorption before reaching the observer, although it may affect the temporal profile of the $2 f_{\mathrm{p}}$ flux (Riddle 1974). As the beam propagates further, $T_{\mathrm{i}}$ decreases, leading to relatively stronger coupling between the primary and product $L$ waves, and thus stronger forward $2 f_{\mathrm{p}}$ emission and higher values of $\mathcal{F}$. Once the beam passes the disturbed region, the source waves and remote radiation regain the properties for case $\mathcal{A}$.

We also found (not shown) for case $C$ that the flux of $f_{\mathrm{p}}$ emission is significantly enhanced ( $\lesssim 1 \mathrm{sfu})$ above the thermal level and may be observable as a microburst (Kundu et al. 1986) at frequencies corresponding to the disturbance. This occurs mainly because the $S$ waves are stronger and the $f_{\mathrm{p}}$ emission rate is higher for higher $T_{\mathrm{i}}$.

Finally, Figs. $3 \mathrm{~d}$ and 4 (blue curve) show the $2 f_{\mathrm{p}}$ spectrum and $\mathcal{F}$ versus $f$, respectively, for case $\mathcal{D}$. We see that the spectrum is repeatedly modulated, each modulation resembling that in Fig. 3b. Furthermore, the variations in $\mathcal{F}$ versus $f$ clearly show the same signature for each $T_{\mathrm{e}}$ disturbance as found for case $\mathcal{B}$. Thus, the signature of $\mathcal{F}$ variations with $f$ for a single $T_{\mathrm{e}}$ disturbance is robust. Figure 4 also shows that greater disturbances in $T_{\mathrm{e}}$ relative to the background lead to qualitatively greater modulations in $\mathcal{F}$ (cf., the peak $\mathcal{F}$ values for the first and third $T_{\mathrm{e}}$ disturbances). Similar results are found for multiple $T_{\mathrm{i}}$ disturbances (not shown).

\section{Discussion and conclusions}

We have developed the first simulations to study the effects on coronal type IIIs of spatially localized disturbances in $T_{\mathrm{e}}$ or $T_{\mathrm{i}}$. The disturbances may be produced by various coronal activities, so the simulations are more realistic than previous work.

The simulations demonstrate that the dynamic spectrum and the curve of maximum flux $\mathcal{F}$ versus frequency $f$ for
$2 f_{\mathrm{p}}$ emission are modulated by the $T_{\mathrm{e}}$ or $T_{\mathrm{i}}$ disturbances. Crucially, the modulation fine structures differ for $T_{\mathrm{e}}$ and $T_{\mathrm{i}}$ disturbances, both qualitatively and quantitatively. A localized increase in $T_{\mathrm{e}}$ produces a signature of "trough $\rightarrow$ peak" in the $\mathcal{F}$ versus $f$ curve, while a "peak $\rightarrow$ trough $\rightarrow$ peak" signature occurs for a localized increase in $T_{\mathrm{i}}$. The different signatures are robust and independent of the details of the disturbances. Thus, localized $T_{\mathrm{e}}$ and $T_{\mathrm{i}}$ disturbances leave identifiable imprints on $2 f_{\mathrm{p}}$ emission. In addition, weak $f_{\mathrm{p}}$ emission may be observable for sufficiently large $T_{\mathrm{i}}$ disturbances. One implication is that the detailed frequency fine structures in coronal type IIIs can be used to probe and differentiate between the localized profiles of $T_{\mathrm{e}}$ and $T_{\mathrm{i}}$. Thus nonthermal coronal type IIIs offer a new tool to remotely diagnose spatial temperature structures in the corona.

We also found that larger disturbances in $T_{\mathrm{e}}$ or $T_{\mathrm{i}}$ relative to the background results in qualitatively larger amplitude modulations in $\mathcal{F}$. Furthermore, for the same disturbance in temperature the modulation effects on $\mathcal{F}$ are more pronounced for $T_{\mathrm{e}}$ than for $T_{\mathrm{i}}$, as suggested by Fig. 4.

The modulations in $2 f_{\mathrm{p}}$ spectrum caused by multiple $T_{\mathrm{e}}$ or $T_{\mathrm{i}}$ disturbances resemble qualitatively the fine structures in type IIIb bursts. Thus, our results suggest that localized disturbances in $T_{\mathrm{e}}$ or $T_{\mathrm{i}}$ may produce type IIIs with striae and modulations in $f$. This mechanism differs from those previously proposed, e.g., modulational instability (Smith \& de La Noe 1976) or filamentary density structures (Takakura \& Yousef 1975).

The flux modulation mechanism here may also apply to type III beams that cross shocks. MacDowall (1989) observed that the flux of IP type IIIs changed suddenly as beams neared shocks. He suggested that the changes are caused by the scattering of beam electrons by magnetic turbulence. We propose, instead, that the heating of electrons and ions just downstream of the shocks produces localized increases in $T_{\mathrm{i}}$ and $T_{\mathrm{e}}$, and so modulations in flux.

Acknowledgements. The Australian Research Council supported this work.

\section{References}

Allen, C. W. 1947, MNRAS, 107, 426

Aschwanden, M. J. 2002, Space Sci. Rev., 101, 1

Aschwanden, M. J. 2006, Physics of the Solar Corona: An Introduction with Problems and Solutions (Springer, Berlin), Chapter 15

Baumbach, S. 1937, Astron. Nachr., 263, 131

Cairns, I. H., Lobzin, V. V., Warmuth, A., et al. 2009, ApJ, 706, L265

de La Noe, J., \& Boischot, A. 1972, A\&A, 20, 55

Fomichev, V. V., \& Chertok, I. M. 1977, Radiophys. Quantum Electron., 20, 869

Klein, K.-L., Krucker, S., Trottet, G., \& Hoang, S. 2005, A\&A, 431, 1047

Kohl, J. L., Strachan, L., \& Gardner, L. D. 1996, ApJ, 465, L141

Kundu, M. R., Gergely, T. E., Szabo, A., Loiacono, R., \& White, S. M. 1986, ApJ, 308, 436

Li, B., Robinson, P. A., \& Cairns, I. H. 2002, Phys. Plasmas, 9, 2976

Li, B., Willes, A. J., Robinson, P. A., \& Cairns, I. H. 2003, Phys. Plasmas, 10, 2748

Li, B., Willes, A. J., Robinson, P. A., \& Cairns, I. H. 2005, Phys. Plasmas, 12 , 012103; 052324

Li, B., Cairns, I. H., \& Robinson, P. A. 2008a, J. Geophys. Res., 113, A06104, A06105

Li, B., Robinson, P. A., \& Cairns, I. H. 2008b, J. Geophys. Res., A10101

MacDowall, R. J. 1989, Geophys. Res. Lett., 16, 923

Parker, E. N. 2007, in Handbook of the Solar-Terrestrial Environment, ed. Y. Kamide, \& A. Chian (Springer, Berlin), 95

Riddle, A. C. 1974, Sol. Phys., 35, 153

Robinson, P. A., \& Benz, A. O. 2000, Sol. Phys., 194, 345

Smith, R. A., \& de La Noe, J. 1976, ApJ, 207, 605

Suzuki, S., \& Dulk, G. A. 1985, in Solar Radiophysics, ed. D. J. McLean, \&

N. R. Labrum (Cambridge: Cambridge Univ. Press), 289

Takakura, T., \& Yousef, S. 1975, Sol. Phys., 40, 421 